\title{
Etude de l'influence de différents paramètres sur la solubilité in vitro de composés industriels ou diuranates d'ammonium calcinés
}

\author{
E. ANSOBORLO, J. CHALABREYSSE, S. ESCALLON * \\ (Manuscrit reçu le 25 juillet 1988)
}

\begin{abstract}
RÉSUMÉ
Le diuranate d'ammonium calciné qui est un mélange de $\mathrm{U}_{3} \mathrm{O}_{8} / \mathrm{UO}_{\mathrm{x}}(2<\mathrm{x}<3)$ en fonction de l'origine du minérai et de la température de calcination est un composé important dans le cycle de l'uranium. Ses caractéristiques physico-chimiques ainsi que sa solubilité ne sont pas toujours bien définies et nous ont conduits à mener cette étude de solubilité in vitro. Deux tests de solubilité in vitro ont été utilisés, à savoir un test dynamique et un test statique avec divers liquides de solubilisation et ce sur deux composés dénommés QML et NUFCOR. Ces tests ont pour but de classer un composé suivant les classes $D, W$ ou $Y$ de la CIPR et de mettre en évidence les paramètres influant sur la solubilité et donc les plus représentatifs des modèles in vivo et humains.

Les résultats obtenus ont confirmé la différence entre ces deux diuranates constatée avec les spectres rayons $\mathrm{X}$, et montré qu'en présence de bicarbonates et de Gamble $+\mathrm{O}_{2}$ la solubilité était plus importante pour NUFCOR que pour QML avec appartenance aux classes $D, W$ pour ces deux composés. Par contre, les phosphates et Gamble constituent une étape limitante de la cinétique avec une tendance $Y$ pour QML et NUFCOR. L'homogénéité des tests conduit à choisir le test statique plus facile à mettre en œuvre et les résultats obtenus avec les différentes solutions devront être validés par des tests in vivo.
\end{abstract}

\section{ABSTRACT}

Calcinated ammonium diuranate - a compound of the uranium industry - may occur as mixture of uranium oxides as $\mathrm{U}_{3} \mathrm{O}_{8} / \mathrm{UO}_{\mathrm{x}}(2<\mathrm{x}<3)$ depending on the source of ore and its thermal conversion. Both its physico-chemical properties and solubllity are not always very well defined and led us to conduct in vitro dissolution experiments. Two kind of tests were used: a flowing and a static system with different fluids on two different calcinated ADU called QML and NUFCOR. These tests were developed in order to classify a compound following the $\mathrm{D}, \mathrm{W}$ or $\mathrm{Y}$ classes of ICRP and also to show up the parameters close to in vivo experiments.

From the results obtained, it appears that both compounds, which have different $X$-ray spectra, have also different solubilities. Their solubilities increase with bicarbonate and Gamble $+\mathrm{O}_{2}$ and are around classes D, W for both compounds, NUFCOR being more soluble than QML. Besides, phosphate and Gamble seem to be a limiting step with $Y$ tendency for QML and NUFCOR. Good recovery of the two in vitro dissolution tests led us to choose the static system which is easier to set up, and results obtained with the different fluids will be validated with in vivo experiments.

* Commissariat à l'énergie atomique, IPSN, Département de protection sanitaire, Service d'hygiène industrielle, BP 38, 26701 Pierrelatte Cedex. 


\section{INTRODUCTION}

La calcination du diuranate d'ammonium est une étape du cycle de traitement de l'uranium pratiquée à la mine après extraction du minerai. Cette opération se fait suivant la réaction:

$$
\left(\mathrm{NH}_{4}\right)_{2} \quad \mathrm{U}_{2} \mathrm{O}_{7} \stackrel{\mathrm{T}}{\rightarrow} \mathrm{U}_{3} \mathrm{O}_{8}+\mathrm{UO}_{x}
$$

Le produit obtenu ou diuranate calciné est en principe de I' $\mathrm{U}_{3} \mathrm{O}_{8}$ avec présence possible, suivant l'origine du minerai et la temperature de calcination, de composés de type $\mathrm{UO}_{\mathrm{x}}$ avec $2<\mathrm{x}<3$.

II est réceptionné à l'usine Comurhex de Narbonne à l'atelier échantillonnage où on le retrouve aux postes de travail suivants: basculement, ensachage et pesée. Se présentant sous forme de poussière, il peut donc être inhalé par le travailleur. Les caractéristiques physico-chimiques et la solubilité de ce composé sont deux points importants pour la protection des personnes exposées.

Afin de prédire le comportement des composés industriels au niveau pulmonaire (classement $\mathrm{D}, \mathrm{W}$ ou $\mathrm{Y}$ ) de la Commission internationale de protection radiologique (CIPR) [10], des tests de solubilité in vitro, faisant intervenir différents types de solutions synthétiques simulant les liquides pulmonaires sont développés depuis quelques années. Ils sont comparés, le plus souvent, à des essais sur cultures cellulaires (macrophages alvéolaires) ou à des expérimentations animales sur rats ou singes décrites par André [1], Kanapilly [13], Damon [4].

Ces essais in vitro permettent une approche des phénomènes chimiques mis en jeu et présentent en outre l'avantage d'être faciles à mettre en œuvre à moindre coût. Mais il est clair que ces tests doivent être validés par des essais in vivo; ils permettent, néanmoins, dans un premier temps, de dégrossir le problème de la classification de composés présents sous forme de mélanges.

Le but de cette étude est double: d'une part classer le diuranate calciné selon la classification de la CIPR et d'autre part développer les tests in vitro suivant deux voies: test dynamique de Kanapilly [12] et test statique de Kalkwarf [11] en utilisant plusieurs solutions de solubilisation afin de comprendre les phénomènes chimiques mis en jeu.

Les solutions de solubilisation retenues sont Gamble [8], Gamble additionnée de $\mathrm{O}_{2}$ ou de $\mathrm{CO}_{2}$ simulant ainsi la présence des gaz inspirés dans le liquide pulmonaire, ou d'eau oxygénée $\mathrm{H}_{2} \mathrm{O}_{2}$ car selon Meyer [14] l'aptitude des macrophages à tuer les germes phagocytés est particulièrement liée à la synthèse d' $\mathrm{H}_{2} \mathrm{O}_{2}$ et de radicaux superoxydes. Ont été également testées une solution de bicarbonates à $3.10^{-3} \mathrm{M} \mathrm{I}^{-1}$, une solution de phosphates à $10^{-3} \mathrm{M} \mathrm{I}^{-1}$ et de l'eau distillée, trois éléments entrant dans la composition de Gamble.

Des résultats concernant le diuranate d'ammonium calciné ont déjà été obtenus par Kalkwarf [11] mettant en évidence que ce composé était $5 \%$ D 
et $95 \%$ Y. D'autres auteurs comme Eidson [6, 7], Damon [4], Denis [5] et Cooke [3] ont étudié le diuranate d'ammonium non calciné et les mélanges $\mathrm{U}_{3} \mathrm{O}_{8}-\mathrm{UO}_{3}$.

Rappelons, enfin, les travaux de Pasquier [16] et Hodge [9] sur l'influence de la valence de l'uranium et les phénomènes de complexation, ainsi que ceux de Moss [15] et Gamble [8] sur les compositions des divers liquides interstitiels.

\section{MÉTHODES ET MATÉRIEL}

\section{Échantillons}

Les deux composés étudiés sont des diuranates d'ammonium calcinés provenant de l'usine de Comurhex Malvési à Narbonne, dénommés QML (en provenance d'Australie) et NUFCOR (en provenance d'Afrique du Sud).

Les caractéristiques physico-chimiques de ces deux composés ont été étudiées: la granulométrie (par Impacteur à cascade Andersen Mark II), la densité (par picnomètre), la surface spécifique (selon la méthode BET) et la composition par rayons $X$ (tableau $\mathrm{I}$ ).

\section{TABLEAU ।}

Caractéristiques physico-chimiques des deux composés QML et NUFCOR

\begin{tabular}{|c|c|c|c|c|}
\hline Composés & $\begin{array}{c}\text { Granulométrie } \\
\text { Dae }(\mu \mathrm{m})\end{array}$ & $\begin{array}{l}\text { Densité } \\
\left(\mathrm{g} \mathrm{cm}^{-3}\right)\end{array}$ & $\begin{array}{l}\text { Surface spécifique } \\
\qquad\left(\mathrm{m}^{2} \mathrm{~g}^{-1}\right)\end{array}$ & $\underset{\mathrm{RX}}{\text { Composition }}$ \\
\hline QML & $\left(^{\star}\right) \stackrel{8 \mathrm{gm}}{\mu \mathrm{m}}=2$ & 8,6 & 6 & $\mathrm{U}_{3} \mathrm{O}_{8}$ \\
\hline NUFCOR & (*) $\begin{array}{l}9 \mu \mathrm{m} \\
\sigma \mathrm{g}=2,2\end{array}$ & 6 & 23,4 & ${ }_{2}^{\mathrm{U}_{3} \mathrm{O}_{8}}+\underset{x}{\mathrm{U}}<\mathrm{U}_{\mathrm{x}}$ \\
\hline
\end{tabular}

(*) $\sigma \mathrm{g}=$ déviation géométrique standard.

Tests in vitro

Deux types de tests ont été retenus:

- Test dynamique ou test à flux parallèle selon Kanapilly [12].

Nous utilisons une chambre en lucite. L'échantillon de poussière est déposé sur un filtre (Millipore GS $-0.22 \mu \mathrm{m}$ ) et recouvert d'un filtre transparent (membrane Cuprophane) l'ensemble formant ainsi un "sandwich" inséré dans la cellule. La quantité de poussière est de l'ordre de 25 à $30 \mathrm{mg}$ et la surface de contact poussière-filtre est mesurée. La circulation 
du liquide de solubilisation est assurée par une pompe péristaltique (ISMATEC MP $13 \mathrm{GP} 4$ ) avec un débit de $0,1 \mathrm{ml} \mathrm{min}^{-1}$ proposé par Kanapilly [13]. Nous avons expérimenté sept solutions différentes à $\mathrm{pH}=7,3$ : Gamble (tableau II), Gamble $+\mathrm{O}_{2}$ (barbotage direct), Gamble $+\mathrm{H}_{2} \mathrm{O}_{2}(0,1$ $\mathrm{cm}^{3}$ à 110 volumes pour $5 \mathrm{~cm}^{3}$ ), une solution de bicarbonates $\mathrm{NaHCO}_{3}$ à $3.10^{-3} \mathrm{M} \mathrm{I}^{-1}$ et une solution de phosphates $\mathrm{Na}_{2} \mathrm{HPO}_{4}$ à $10^{-3} \mathrm{M} \mathrm{I}^{-1}$ (concentrations équivalentes à celles de Gamble) et de l'eau distillée.

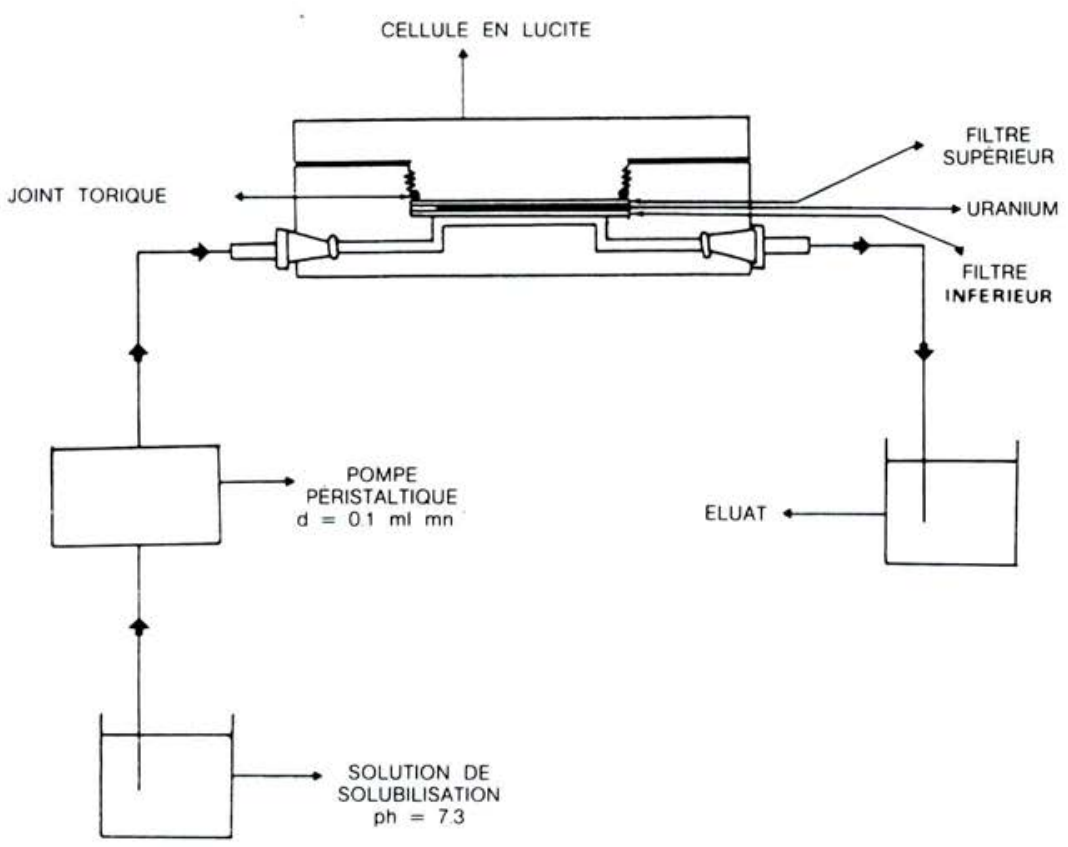

Fig. 1. - Système de dissolution in vitro dynamique.

TABLEAU ॥

Composition de la solution de Gamble

\begin{tabular}{|c|c|c|c|c|c|c|c|c|}
\hline Composé & $\mathrm{NaCl}$ & $\mathrm{NH}_{4} \mathrm{Cl}$ & $\mathrm{NaHCO}_{3}$ & $\mathrm{NaH}_{2} \mathrm{PO}_{4}$ & $\begin{array}{c}\text { citrate } \\
\text { trisodique }\end{array}$ & glycine & cystéine & $\mathrm{CaCl}_{2}$ \\
\hline $\begin{array}{c}\text { Concentration } \\
\text { (mmole I }\end{array}$ & 116 & 10 & 27 & 1,2 & 0,2 & 6 & 1 & 0,2 \\
\hline
\end{tabular}

- Test statique pratiqué selon Kalkwarf [11].

Ce test est pratiqué dans deux types de récipients en verre: pour les solutions sans barbotage, nous utilisons le modèle de Kalkwarf [11] avec 
agitateur magnétique (fig. 2). La quantité de poussière déposée est de 6 à $10 \mathrm{mg}$ dans une fiole conique de $5 \mathrm{~cm}^{3}$.

Pour la solution avec barbotage $\left(\mathrm{O}_{2}\right)$ nous travaillons avec des tubes en verre de $10 \mathrm{~cm}^{3}$ dans lesquels plonge le tube de bullage et les quantités de poussières sont de l'ordre de $15 \mathrm{mg}$.

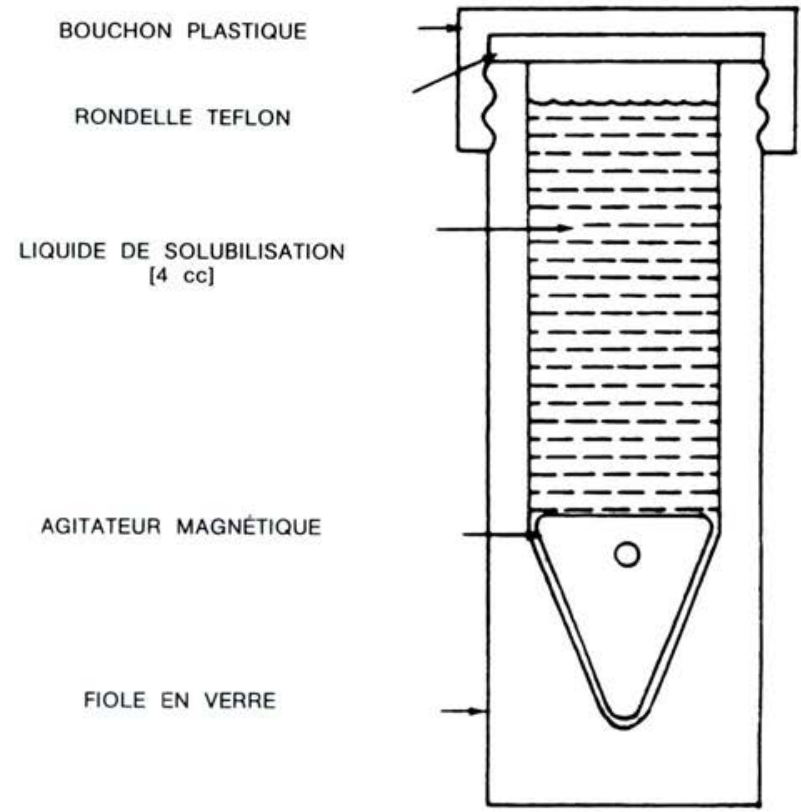

Fig. 2. - Système de dissolution in vitro statique.

\section{Analyses et expression des résultats}

Le dosage de l'uranium dans les échantillons s'effectue par fluorimétrie à l'aide d'un appareil à fluorescence $X$ Schlumberger (modèle CEA). Les résultats sont exprimés en pourcentage d'uranium cumulé non dissous en fonction du temps.

Le calcul consiste en un ajustement par régression non linéaire des moindres carrés et les résultats sont obtenus sous forme de sommes d'exponentielles:

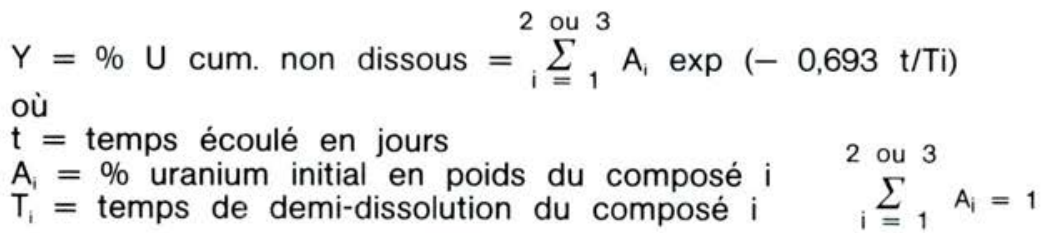




\section{RÉSULTATS}

Les résultats sont reportés sur le tableau III et sont exprimés, pour chacun des deux composés QML et NUFCOR, en terme de $A_{i}$ et $T_{i}$ definis ci-dessus pour chaque classe D, W, Y de la CIPR [10].

\section{TABLEAU III}

Solubilité des composés QML et NUFCOR en essai statique et dynamique avec diverses solutions

\begin{tabular}{|c|c|c|c|c|c|c|c|c|c|}
\hline \multirow{3}{*}{$\begin{array}{c}\text { Liquides } \\
\text { de } \\
\text { solubilité }\end{array}$} & \multirow{3}{*}{$\begin{array}{l}\text { Classes } \\
\text { (CIPR) }\end{array}$} & \multicolumn{4}{|c|}{ QML $\left(\mathrm{U}_{3} \mathrm{O}_{8}\right)$} & \multicolumn{4}{|c|}{ NUFCOR $\left(\mathrm{U}_{3} \mathrm{O}_{8}+\mathrm{UO}_{\mathrm{x}}\right)$} \\
\hline & & \multicolumn{2}{|c|}{$\left({ }^{*}\right) A_{i}(\%)$} & \multicolumn{2}{|c|}{${ }^{(*)} \mathrm{T}_{\mathrm{i}}(\mathrm{j})$} & \multicolumn{2}{|c|}{$\left({ }^{*}\right) A_{i}(\%)$} & \multicolumn{2}{|c|}{$\left({ }^{\star}\right) T_{i}(j)$} \\
\hline & & Stat. & Dyna. & Stat. & Dyna. & Stat. & Dyna. & Stat. & Dyna. \\
\hline $\mathrm{NaHCO}_{3}$ & $\begin{array}{l}\text { D } \\
\text { W }\end{array}$ & $\begin{array}{l}19,2 \\
80,8\end{array}$ & $\begin{array}{l}21 \\
79\end{array}$ & $37^{0,4}$ & $\begin{array}{r}1 \\
58\end{array}$ & 100 & $\begin{array}{l}65 \\
35\end{array}$ & 3,5 & $\begin{array}{l}0,8 \\
21\end{array}$ \\
\hline $\mathrm{NaH}_{2} \mathrm{PO}_{4}$ & $\begin{array}{l}\mathrm{D} \\
\mathrm{W} \\
\mathrm{Y}\end{array}$ & 100 & 100 & 450 & 690 & $\begin{array}{l}11,3 \\
88,7\end{array}$ & 100 & $\begin{array}{r}1 \\
485\end{array}$ & 775 \\
\hline $\mathrm{H}_{2} \mathrm{O}$ & $\begin{array}{l}D \\
W \\
Y\end{array}$ & $\begin{array}{l}11,3 \\
88,7\end{array}$ & 100 & $\begin{array}{r}0,3 \\
358\end{array}$ & 476 & $\begin{array}{l}40 \\
60\end{array}$ & $\begin{array}{l}25,5 \\
74,5\end{array}$ & $\begin{array}{r}0,1 \\
224\end{array}$ & $\begin{array}{r}0,3 \\
277\end{array}$ \\
\hline Gamble & $\begin{array}{l}D \\
W \\
Y\end{array}$ & $\begin{array}{r}5,1 \\
94,9\end{array}$ & $\begin{array}{r}5,5 \\
94,5\end{array}$ & $\begin{array}{r}0,6 \\
1024\end{array}$ & $\begin{array}{r}2 \\
1110\end{array}$ & $\begin{array}{l}14,6 \\
85,4\end{array}$ & $\begin{array}{r}8,5 \\
91,5\end{array}$ & $\begin{array}{r}0,5 \\
559\end{array}$ & $\begin{array}{r}1 \\
387\end{array}$ \\
\hline Gamble $+\mathrm{O}_{2}$ & $\begin{array}{l}D \\
W \\
Y\end{array}$ & $\begin{array}{l}19,5 \\
80,5\end{array}$ & $\begin{array}{r}6 \\
94\end{array}$ & $\begin{array}{l}0,3 \\
31\end{array}$ & $\begin{array}{r}2 \\
407\end{array}$ & $\begin{array}{l}35,2 \\
64,8\end{array}$ & $\begin{array}{l}38,6 \\
61,4\end{array}$ & $\begin{array}{l}0,3 \\
15\end{array}$ & $\begin{array}{r}0,8 \\
186\end{array}$ \\
\hline$\underset{\mathrm{H}_{2} \mathrm{O}_{2}}{\text { Gamble }}+$ & $\begin{array}{l}D \\
W \\
Y\end{array}$ & $\begin{array}{l}14,8 \\
85,2\end{array}$ & & $\begin{array}{l}0,8 \\
60^{0}\end{array}$ & & $\begin{array}{l}21,4 \\
78,6\end{array}$ & & $\begin{array}{r}0,3 \\
150\end{array}$ & \\
\hline
\end{tabular}
(*) Le \% U non dissous est exprimé comme $\sum_{i=1}^{2}$ ou ${ }_{i}^{3} A_{i} \exp \left(-0.693 t / T_{i}\right)$
avec :
$\mathrm{A}_{\mathrm{i}} \quad=\%$ uranium initial en poids du composé $\mathrm{i}$
$\mathrm{T}_{\mathrm{i}} \quad=$ temps de demi-dissolution du composé $\mathrm{i}$
$\mathrm{D}, \mathrm{W}, \mathrm{Y}=$ classes de la CIPR 


\section{DISCUSSION}

\section{Comparaison des deux tests:}

Le tableau III traduit une bonne homogénéité entre les essais statiques et dynamiques pour chacune des solutions étudiées. Ceci est intéressant car la mise en œuvre des essais statiques est beaucoup plus aisée que celle des essais dynamiques (préparation des échantillons, débit, conservation des solutions).

Pour les deux composés étudiés (QML et NUFCOR) les résultats sont reproductibles pour les quatre solutions $\left(\mathrm{NaHCO}_{3}, \mathrm{Na}_{2} \mathrm{HPO}_{4}\right.$, eau, Gamble). On note une différence au niveau des barbotages directs: le degré de solubilisation observé en dynamique est plus faible qu'en statique. Ceci semble logique puisque, pour un bullage d'O $\mathrm{O}_{2}$ équivalent, l'enrichissement en $\mathrm{O}_{2}$ est beaucoup plus important en statique du fait que le volume de solubilisation est plus faible.

Notons que le composé NUFCOR identifié comme $\mathrm{U}_{3} \mathrm{O}_{8}+\mathrm{UO}_{\mathrm{x}}(2<\mathrm{x}<3)$ quelle que soit la configuration expérimentale est plus soluble que le composé $\mathrm{QML}$ identifié comme $\mathrm{U}_{3} \mathrm{O}_{8}$.

\section{Comparaison des effets sur la solubilité des diverses solutions utilisées.}

Nous avons pensé qu'il était intéressant d'analyser l'influence des divers constituants de Gamble (notamment $\mathrm{HCO}_{3}{ }^{-}$et $\mathrm{HPO}_{4}{ }^{2}$ ), d'un apport gazeux par barbotage $\left(\mathrm{O}_{2}\right)$ ou $\left(\mathrm{CO}_{2}\right)$ ainsi que le rôle éventuel de l'eau oxygénée $\left(\mathrm{H}_{2} \mathrm{O}_{2}\right)$.

Pour le composé $\mathrm{QML}\left(\mathrm{U}_{3} \mathrm{O}_{8}\right)$, nous observons que les bicarbonates favorisent la solubilisation aussi bien en dynamique qu'en statique avec trois résultats concordant autour de $20 \% \mathrm{D}-80 \% \mathrm{~W}$ (résultat déjà noté lors d'une étude d'Ansoborlo [2] sur $\mathrm{UF}_{4}$ ).

Les phosphates qui à $\mathrm{pH}=7,3$ sont aussi bien sous forme $\mathrm{HPO}_{4}{ }^{=}$que $\mathrm{H}_{2} \mathrm{PO}_{4}{ }^{-}$semblent être un élément limitant puisqu'en dynamique comme en statique $\mathrm{QML}$ a un comportement de type $\mathrm{Y}$.

Pour la solution de Gamble, on note une assez bonne homogénéité des résultats avec en moyenne $3 \% \mathrm{D}$ et $97 \% \mathrm{Y}$. Ce comportement $\mathrm{Y}$ de QML correspond bien à la classification de la CIPR et aux résultats obtenus par Kalkwarf [11], Cooke [3] et Eidson [6, 7] sur $\mathrm{U}_{3} \mathrm{O}_{8}$.

II apparaît ainsi que l'effet limitant sur la solubilité de la solution de Gamble soit lié aux phosphates présents, qui inhibent l'action des carbonates. L'addition d'oxygène à Gamble par barbotage direct en statique augmente la solubilité avec $20 \%$ D - $80 \%$ W. Enfin, l'addition d'eau oxygénée à Gamble fournit un résultat intéressant avec $15 \% \mathrm{D}-85 \% \mathrm{~W}$. $\mathrm{Ce}$ résultat est très proche de ceux obtenus avec Gamble $+\mathrm{O}_{2}$ et les bicarbonates. 
Pour le composé NUFCOR $\left(\mathrm{U}_{3} \mathrm{O}_{8}+\mathrm{UO}_{\mathrm{x}}(2<\mathrm{x}<3)\right)$ les bicarbonates favorisent la solubilisation avec une tendance $D$. En présence de phosphates NUFCOR est plutôt insoluble de tendance $\mathrm{Y}$.

Dans Gamble, on note une assez bonne homogénéité des résultats avec $10 \% \mathrm{D}-90 \%$ Y, ce qui confirme l'aspect inhibiteur des phosphates. L'oxygène additionnée à Gamble en statique conduit à $35 \% \mathrm{D}-65 \% \mathrm{~W}$, soit un accroissement de la solubilité. Enfin l'addition d' $\mathrm{H}_{2} \mathrm{O}_{2}$ à Gamble a donné des résultats surprenants avec une réaction puissante sur NUFCOR, accompagnée d'un dégazage au niveau de la poussière et d'un changement de couleur de marron noir à jaune vif identifié aux rayons $X$ comme $\cup_{4}, 3 \mathrm{H}_{2} \mathrm{O}$. Le composé obtenu semble toujours peu soluble avec $21 \% \mathrm{D}-97 \%$ Y mais, toutefois, plus soluble que dans la solution de Gamble seule puisque la période $\mathrm{T}_{\mathrm{i}}$, tout en restant classe $\mathrm{Y}$, passe de $559 \mathrm{j}$ à $150 \mathrm{j}$.

\section{CONCLUSIONS}

- En présence des solutions $\mathrm{NaHCO}_{3}, \mathrm{Na}_{2} \mathrm{HPO}_{4}, \mathrm{H}_{2} \mathrm{O}$ et Gamble les résultats obtenus par les deux tests sont homogènes, malgré des quantités de poussières différentes; le test statique, plus aisé de mise en œuvre, est le plus intéressant à utiliser.

- L'apport gazeux d'oxygène à Gamble augmente de façon notoire la solubilité des deux composés QML et NUFCOR: ils deviennent $D, W$ dans l'essai statique avec barbotage. Des essais pratiqués avec $\mathrm{CO}_{2}$ par barbotage dans Gamble ont donné des résultats identiques à Gamble, montrant ainsi que cet apport gazeux n'intervenait pas dans la solubilité de ces deux composés. L'essai statique paraît plus intéressant à utiliser que le dynamique car il y a contact direct entre la poussière et le mélange gaz-solution.

- L'addition d'eau oxygénée $\mathrm{H}_{2} \mathrm{O}_{2}$ a mis en évidence pour QML une solubilisation accrue, identique à celle observée pour les bicarbonates et Gamble $+\mathrm{O}_{2}$. Par contre, pour NUFCOR, nous avons noté une transformation chimique rendant le composé un peu plus soluble (20 \% D - $80 \%$ Y) comparativement aux résultats obtenus avec la solution de Gamble.

- Les solutions qui semblent devoir être retenues sont: les bicarbonates, Gamble, Gamble $+\mathrm{O}_{2}$ et Gamble $+\mathrm{H}_{2} \mathrm{O}_{2}$. En effet, les phosphates qui constituent l'étape limitante se trouvent dans Gamble, l'eau n'apporte pas d'élément supplémentaire et le $\mathrm{CO}_{2}$ n'a pas d'effet.

- II apparaît que les deux composés QML et NUFCOR dont la composition chimique ne semblait pas trop éloignée se comportent différemment. Dans l'ensemble NUFCOR est toujours plus soluble que QML, ce qui tend à prouver que le composé $\mathrm{UO}_{\mathrm{x}}$ est un composé assez soluble.

- Nous avons mis en évidence l'influence importante des bicarbonates (augmentation de la solubilité), des phosphates (formation des complexes insolubles) et de l'oxygène additionnée à Gamble (augmentation de la solubilité). 
Certains essais préliminaires in vivo par inhalation et instillation sur rats ont montré une tendance $\mathrm{D}, \mathrm{W}$ du composé NUFCOR.

L'étape suivante de cette étude consistera en la validation et l'étalonnage des essais in vitro par comparaison d'une part avec un test in vitro cellulaire développé par Métivier [1] et d'autre part avec deux tests in vivo chez le rat, à savoir un dépôt intratrachéal et une inhalation de ces deux composés.

\section{REMERCIEMENTS}

Nous remercions tout particulièrement M. BLANCHIN et le Dr GIBERT, de l'usine Comurhex Malvési à Narbonne, pour leur contribution à cette étude, initiée par le regretté Dr CAMARASA.

\section{BIBLIOGRAPHIE}

[1] ANDRÉ S., METIVIER H. - Beryllium metal solubility in the lung, Comparison of metal and hot-pressed forms by in vivo and in vitro dissolution bioassays. Human Toxicol., 1987, 6, 233-240.

[2] ANSOBORLO E. - Etude des caractéristiques physico-chimiques de composés uranifères sur des postes de travail. Thèse de Docteur Ingénieur, ECAM, 1983.

[3] COOKE N., HOLT F.B. - The solubility of some uranium compounds in simulated lung fluid. Health Phys., 1974, 27, 69-77.

[4] DAMON E.G., EIDSON A.F. - Comparison of early lung clearance of yellowcake aerosols in rats with in vitro dissolution and IR analysis. Health Phys., 1984, 46, 859-866.

[5] DENNIS N.A., BLAUER H.M. - Dissolution fractions and half-times of single source yellowcake in simulated lung fluids. Health Phys., 1982, 42, 469-477.

[6] EIDSON A.F., GRIFFITH W.C. - Techniques for yellowcake dissolution studies in vitro and their use in bioassay interpretation. Health Phys., 1984, 46, 151-163.

[7] EIDSON A.F., MEWHINNEY J.A. - In vitro solubility of yellowcake samples from four uranium mills and the implications for bioassay interpretation. Health Phys., $1980,39,893-902$.

[8] GAMBLE J.L. - Chemical anatomy, physiology and pathology of extra cellular fluid, 8th edition. Harvard: Harvard university press, 1967, 4-11.

[9] HODGE H.C., STANNARD J.N. - Uranium, plutonium, transplutonic elements. Heidelberg: Springer-Verlag, 1973, 112-130.

[10] INTERNATIONAL COMMISSION ON RADIOLOGICAL PROTECTION (ICRP) Limits for intakes of radionuclides by workers. ICRP publication 30. Oxford: Pergamon Press, 1979.

[11] KALKWARF D.R. - Solubility classification of airborne uranium products from LWR fuel plants. NUREG/CR-1428 (PNL-3411), 1980.

[12] KANAPILLY G.M., GOH C.H.T. - Some factors affecting the in vitro rates of dissolution of respirable particles of relatively low dissolution. Health phys. 1973, 25, 225-237. 
[13] KANAPILLY G.M., RAABE O.G. - Measurement of in vitro dissolution of aerosol particles for comparison to in vivo dissolution in the lower respiratory tract after inhalation. Health Phys. 1973, 24, 497-507.

[14] MEYER P. - Physiologie humaine. Paris: Flammarion Médecine - Sciences, 1983.

[15] MOSS O. - Simulates of lung intestitial fluid. Health Phys. 1979, 36, 446.

[16] PASQUIER C., BOURGUIGNON M. - Etude expérimentale de la fixation rénale après contamination aiguë par l'uranium - Influence de la solubilité des composés utilisés $\mathrm{UO}_{2}, \mathrm{UO}_{3}, \mathrm{U}_{3} \mathrm{O}_{8}$. In: Biological implications of radionuclides released from nuclear industries. Vienne: AIEA, 1979, 385-399. 